\title{
Asymptotic behavior of a three-species food chain model with time-varying delays
}

\author{
Yuxiao Zhao ${ }^{1}$, Linshan Wang $^{2}$, and Yangfan Wang ${ }^{1}$ \\ ${ }^{1}$ Ocean University of China \\ ${ }^{2}$ Department of Mathematics, Ocean University of China, Qingdao, Shandong 266071, PR \\ China
}

March 13, 2021

\begin{abstract}
In this paper, a stochastic three-species food chain model with time-varying delays is focussed. The existence and the asymptotic behavior of global positive solutions to the model are discussed, and the sufficient conditions for the 1th moment practical exponential stability and the extinction of the model are given by using the Razumikhin technique and Lyapunov method.
\end{abstract}

\section{Hosted file}

wileyNJD-Doc.pdf available at https://authorea.com/users/401386/articles/513483-asymptoticbehavior-of-a-three-species-food-chain-model-with-time-varying-delays 